\title{
High Fibrinogen to Albumin Ratio: A Novel Marker for Risk of Stroke-Associated Pneumonia?
}

OPEN ACCESS

Edited by:

Bo Wu,

West China Hospital of Sichuan

University, China

Reviewed by:

Changyi Wang,

Sichuan University, China

Jianjun Zou,

Nanjing Hospital Affiliated to Nanjing

Medical University, China

María Fernanda Raya Tonetti, Consejo Nacional de Investigaciones

Científicas y Técnicas

(CONICET), Argentina

*Correspondence:

Guiqian Huang

huangguiqian123@126.com

Jincai He

hjc@wmu.edu.cn

Zhen Wang

wangzhen@wzhospital.cn

tThese authors have contributed equally to this work

Specialty section:

This article was submitted to Neurological Biomarkers,

a section of the journal

Frontiers in Neurology

Received: 27 August 2021 Accepted: 14 December 2021

Published: 13 January 2022

Citation:

Lin G, Hu M, Song J, Xu X, Liu H, Qiu L, Zhu H, Xu M, Geng D, Yang L, Huang G, He J and Wang Z (2022) High Fibrinogen to Albumin Ratio: A

Novel Marker for Risk of

Stroke-Associated Pneumonia?

Front. Neurol. 12:747118.

doi: 10.3389/fneur.2021.747118

\author{
Gangqiang Lin ${ }^{1+}$, Minlei Hu${ }^{2 \dagger}$, Jiaying Song ${ }^{3+}$, Xueqian $\mathrm{Xu}{ }^{1}$, Haiwei Liu ${ }^{1}$, Linan Qiu ${ }^{1}$, \\ Hanyu Zhu ${ }^{1}$, Minjie $\mathrm{Xu}^{3}$, Dandan Geng ${ }^{1}$, Lexuan Yang ${ }^{1}$, Guiqian Huang ${ }^{1 *}$, Jincai He ${ }^{1 *}$ and \\ Zhen Wang ${ }^{1 *}$

\begin{abstract}
'Department of Neurology, The First Affiliated Hospital of Wenzhou Medical University, Wenzhou, China, ${ }^{2}$ Department of Neurology, The First Hospital of Jiaxing, Jiaxing, China, ${ }^{3}$ School of Mental Health, Wenzhou Medical University, Wenzhou, China
\end{abstract}

Background: Stroke-associated pneumonia (SAP) is associated with poor prognosis after acute ischemic stroke (AIS).

Purpose: This study aimed to describe the parameters of coagulation function and evaluate the association between the fibrinogen-to-albumin ratio (FAR) and SAP in patients with AIS.

Patients and Methods: A total of 932 consecutive patients with AIS were included. Coagulation parameters were measured at admission. All patients were classified into two groups according to the optimal cutoff FAR point at which the sum of the specificity and sensitivity was highest. Propensity score matching (PSM) was performed to balance potential confounding factors. Univariate and multivariate logistic regression analyses were applied to identify predictors of SAP.

Results: A total of 100 (10.7\%) patients were diagnosed with SAP. The data showed that fibrinogen, FAR, and D-dimer, prothrombin time (PT), activated partial thromboplastin time (aPTT) were higher in patients with SAP, while albumin was much lower. Patients with SAP showed a significantly increased FAR when compared with non-SAP $(P<$ 0.001). Patients were assigned to groups of high FAR $(\geq 0.0977)$ and low FAR $(<0.0977)$ based on the optimal cut-off value. Propensity score matching analysis further confirmed the association between FAR and SAP. After adjusting for confounding and risk factors, multivariate regression analysis showed that the high FAR ( $\geq 0.0977)$ was an independent variable predicting the occurrence of SAP (odds ratio $=2.830,95 \% \mathrm{Cl}=1.654-4.840$, $P<0.001)$. In addition, the FAR was higher in the severe pneumonia group when it was assessed by pneumonia severity index $(P=0.008)$.

Conclusions: High FAR is an independent potential risk factor of SAP, which can help clinicians identify high-risk patients with SAP after AIS.

Keywords: acute ischemic stroke, stroke-associated pneumonia, coagulation, inflammation, fibrinogen, albumin 


\section{INTRODUCTION}

Stroke-associated pneumonia (SAP) is one of the most common complications among patients with acute ischemic stroke (AIS), taking place most frequently within the first week of stroke onset (1-3). The incidence of SAP ranges from 6.7 to $37.98 \%$ (4-6). Patients with SAP are more likely to have a worse outcome than patients with non-SAP, namely, poor functional prognosis, excessive time in hospital, and an increased risk of disability, 30-day and 1-year mortality (7-9). In addition, a retrospective cohort study including 14,702 patients with AIS found that pneumonia was significantly associated with the development of non-pneumonia medical complications, such as gastrointestinal bleeding, urinary tract infection, and recurrent stroke (10). Several clinical trials have shown that prophylactic use of antibiotics was ineffective to prevent SAP $(11,12)$.

Numerous studies have discovered various risk factors for SAP such as old age, being male, stroke severity, dysphagia, and diabetes $(5,9,13)$. Moreover, researchers created several predictive models with these risk factors $(4,14-17)$, among which the A2DS2 score [age, atrial fibrillation (AF), dysphagia, sex, and severity] was considered to have a good predictive capacity (18). In a consecutive cohort of 1,569 patients with AIS, Gong et al. proved that the A2DS2 score could effectively predict the development of SAP in the Chinese population (19). However, SAP prediction is still a challenge due to its atypical clinical symptoms and the low accuracy of X-ray images (20). Therefore, it is necessary for us to look for valid predictors to diagnose SAP as early as possible.

It has long been known that there exists a wide range of interplay between inflammation and coagulation. For instance, inflammation spikes immediately after injury. Aggregated platelets and neutrophils release factors that stimulate the coagulation cascade in acute lung disease (21).

The fibrinogen-to-albumin ratio (FAR) which combines coagulation with nutritional status, is a new vital inflammatory biomarker for a variety of diseases, such as cervical cancer, oligodendroglial gliomas, acute coronary syndrome, and stroke (22-25). Zheng et al. found that a high FAR level on admission was highly associated with 3 -month mortality and disability in patients with acute lacunar stroke (22). Yet, the link between FAR and the risk of developing SAP in patients after AIS remains unclear. The pneumonia severity index (PSI) is one of the best-known predictors for prognosis, containing 20 variables covering demography, clinical features, physical examination, laboratory examination, and chest radiography (26). A decade after PSI was founded, Aujesky et al. proved that PSI could effectively predict mortality and other adverse outcomes of low-risk patients and provided useful feedback to guide initial treatment (27).

Abbreviations: AIS, acute ischemic stroke; PSM, propensity score matching; AUROC, area under the receiver operating characteristic curve; OR, odds ratio; FAR, fibrinogen to albumin ratio; SBP, systolic blood pressure; DBP, diastolic blood pressure; NIHSS, National Institute of Health Stroke Scale; AF, atrial fibrillation; mRS, modified Rankin Scale.
Here, we aimed to explore the relationship between SAP and FAR in a retrospective cohort, which might provide an easily available and economical predictor for SAP.

\section{MATERIALS AND METHODS}

\section{Study Population}

Patients were collected from a retrospective clinical database that included consecutive patients admitted to the Department of Neurology, First Affiliated Hospital of Wenzhou Medical University, within $24 \mathrm{~h}$ after the onset of ischemic stroke between March 2018 and January 2019 in this retrospective study. This retrospective study obtained the approval of the Ethics Committee of the First Affiliated Hospital of Wenzhou Medical University and was conducted according to the ethical standards of the local Research Ethics Committee on human experimentation.

Included patients diagnosed with AIS were verified with CT or MRI within $24 \mathrm{~h}$ at admission. The exclusion criteria were as follows (i) transient ischemic attack; (ii) active infection within 2 weeks before admission or prophylactic use of antibiotics; (iii) a history of central nervous system diseases such as brain trauma, cerebral hemorrhage, or hydrocephalus; (iv) severe liver disease [serum transaminase levels more than twice the upper limit of the normal range or persistent hyperbilirubinemia within 6 months]; (v) severe kidney disease [estimated glomerular filtration rate (eGFR) $<60 \mathrm{ml} / \mathrm{min} / 1.73 \mathrm{~m}^{2}$ ]; and (vi) incomplete medical or laboratory record. Ultimately, a total of 932 patients were enrolled in this study (Figure 1).

\section{Data Collection}

Demographic data included age, gender, history of previous disease, smoking, and drinking. Pre-existing comorbidities included hypertension, diabetes mellitus, coronary artery disease, AF. Other clinical variables collected prescriptions during hospital (antiplatelet, anticoagulation, stain, and thrombolysis), laboratory tests [fibrinogen, platelets, prothrombin time (PT), activated partial thromboplastin time (aPTT), international normalized ratio (INR), D-dimer, and albumin], and blood pressure measurements were conducted within $24 \mathrm{~h}$ of hospital admission. Stroke severity, evaluated using the National Institutes of Health Stroke Scale at admission were assessed by well-trained neurologists. Functional outcomes were assessed by the modified Rankin Scale (mRS) at discharge. The same occupational neurologist evaluated swallowing function in all participants at baseline with the modified water swallowing test. The assessment mainly consisted of behavioral airway response, especially coughing, choking, or throat clearing, and/or change in voice (28). The PSI, a well-verified scoring system of pneumonia burden, was used to assed the severity of pneumonia in the SAP participants (29).

\section{Assessment of Outcome}

Stroke-associated pneumonia was defined as lower respiratory tract infections within the first 7 days after stroke onset, accordance to the modified Center for Disease Control and Prevention criteria (30). Patients were diagnosed with SAP based 


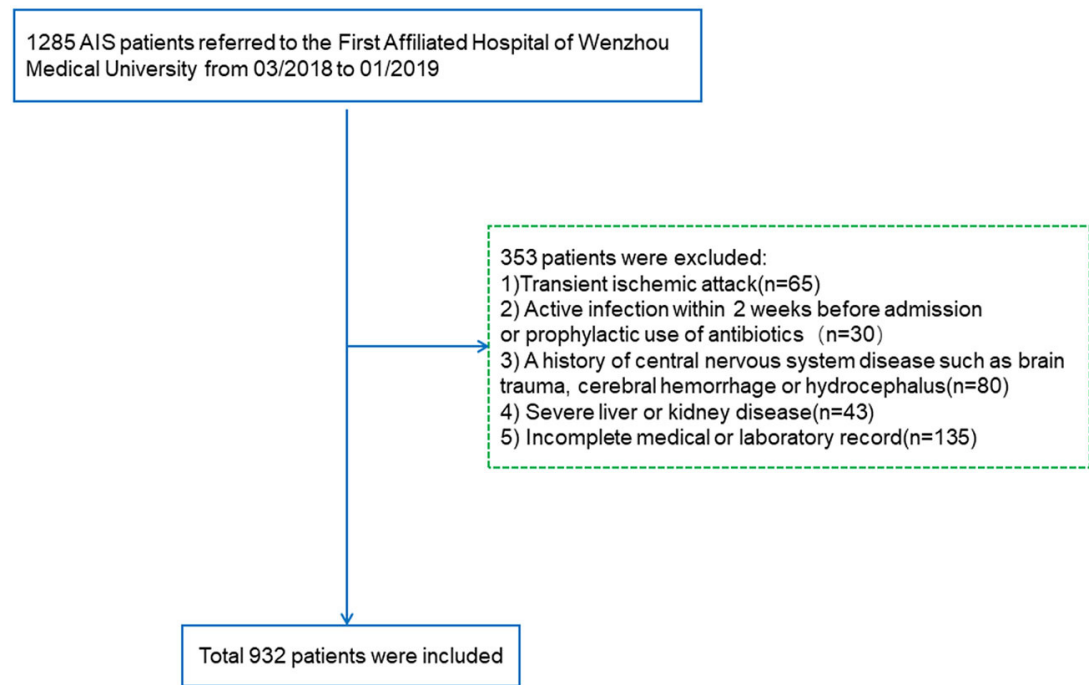

FIGURE 1 | Research flowchart. AIS, acute ischemic stroke.

on clinical symptoms, laboratory examination,_and confirmation by chest X-ray and CT (14). Furthermore, the diagnosis of SAP was conducted by two well-trained neurologists blinded to the data of the patients. This study only recorded in-hospital pneumonia; community-acquired pneumonia and pneumonia before the stroke were excluded from consideration.

\section{Propensity-Score Matching}

We used propensity-score matching to balance confounding factors in baseline characteristics between the low FAR group and high FAR group. The propensity score was estimated using logistic regression of independent variables for each patient that included age, current smoking, drinking, NHISS, previous stroke, diabetes mellitus, AF, thrombolysis, dysphagia, aPTT, D-dimer, PLT, length of hospital stay, and discharge mRS score. If the propensity scores of patients were the same, they had an equal probability of encountering events that we wanted to observe. We adopted 1:1 propensity score-matching to match the low FAR group and high FAR group.

\section{Statistical Analysis}

Continuous variables were presented as mean with standard deviation or median with interquartile range according to normal or asymmetrical distribution, while discrete variables were shown as frequencies or percentages. Data normality was checked with Kolmogorov-Smirnov test. Continuous variables in normal distribution were compared by Student's $t$-test, while asymmetrically distributed variables were analyzed by MannWhitney U-test, and comparison of proportions were analyzed by the chi-squared test. The relationship between FAR and SAP was analyzed in four steps. First, we conducted a receiver operating characteristic (ROC) curve to determine the optimal value of the area under the curve (AUC), and a significant cutoff point, sensitivity, and specificity. We considered the optimal cutoff point by calculating Youden's index max point giving the highest sum of sensitivity and specificity and maximum diagnostic efficiency. Second, binary logistic regression was performed to explore the relationship between this FAR cutoff point and SAP. Third, all confounding factors $(P<0.05)$ were included in multiple logistic regression to adjust for potential confounding factors and find out the independent factors for SAP. Finally, we compared FAR levels between the severe SAP group and non-severe SAP group divided by PSI. All statistical analyses were performed with SPSS for Windows, version 25.0 (SPSS Inc., Chicago, IL, USA). All the statistics are two-tailed and $P<0.05$ was considered statistically significant.

\section{RESULTS}

\section{Baseline of Characteristics of Patients in SAP Group and Non-SAP Group}

During the research period, 932 patients with AIS were included in the study. The baseline demographic, clinical, and laboratory characteristics of the study population are displayed in Table $\mathbf{1 .}$ A total of $593(63.0 \%)$ patients were male, and the mean age of the enrolled patients was 67.0 years (59.0-74.0 years). Compared with patients with non-SAP, SAP patients who were older $(P$ $<0.001)$, had higher National Institute of Health Stroke Scale (NIHSS) scores at admission $(P<0.001)$, higher mRS scores at discharged and longer hospital stay, were more likely to acquire dysphagia $(P=0.031)$. Besides, patients with SAP were more likely to receive anticoagulation treatment and were less likely to undergo antiplatelet therapies.

\section{Comparison of Initial Indexes of Coagulation Function Between Patients With SAP and Non-SAP}

It was shown that fibrinogen, FAR, PT, INR, aPTT, and D-dimer were significantly higher in the SAP group than in the non-SAP 
TABLE 1 | Baseline of characteristics of patients in SAP group and non-SAP group.

\begin{tabular}{|c|c|c|c|c|}
\hline Variables & All patients & Non-SAP $(n=832)$ & SAP $(n=100)$ & $P$ value \\
\hline FAR & 0.089 (0.075-0.109) & $0.087(0.074-0.105)$ & $0.113(0.090-0.167)$ & $<0.001$ \\
\hline Age (years) & $67.0(59.0-74.0)$ & $66.0(59.0-73.0)$ & $72.0(62.5-80.0)$ & $<0.001$ \\
\hline Male & $596(63 \%)$ & $533(64.1 \%)$ & $63(63.0 \%)$ & 0.834 \\
\hline Current smoking & 376 (40.3\%) & 338 (40.6\%) & 38 (38.0\%) & 0.613 \\
\hline Drinking & $350(37.6 \%)$ & $314(37.7 \%)$ & 36 (36.0\%) & 0.734 \\
\hline Baseline SBP & $151.5(136.0-168.0)$ & $151.0(136.0-168.0)$ & $152.0(138.0-168.0)$ & 0.475 \\
\hline Baseline DBP & 82.0 (74.0-92.0) & $82.0(74.0-92.0)$ & $83.0(77.0-94.5)$ & 0.267 \\
\hline NHISS & $3.0(1.0-6.0)$ & $2.0(1.0-5.0)$ & $9.5(3.5-13.0)$ & $<0.001$ \\
\hline Previous stroke & 133 (14.3\%) & 112 (13.5\%) & $21(21.0 \%)$ & 0.042 \\
\hline Hypertension & 713 (76.5\%) & 635 (76.3\%) & 78 (78.0\%) & 0.708 \\
\hline Diabetes mellitus & 369 (39.6\%) & 338 (40.6\%) & 31 (31.0\%) & 0.063 \\
\hline CAD & $20(2.2 \%)$ & $19(2.3 \%)$ & $1(1.0 \%)$ & 0.401 \\
\hline AF & 111 (11.9\%) & $83(10.0 \%)$ & 28 (28.0\%) & $<0.001$ \\
\hline Dysphagia & 129 (13.8\%) & 74 (8.9\%) & 55 (55.0\%) & $<0.001$ \\
\hline Antiplatelet drugs & 883 (93.7\%) & 798 (95.9\%) & 85 (85.0\%) & $<0.001$ \\
\hline Anticoagulant drugs & $129(13.8 \%)$ & 108 (13.0\%) & $21(21.0 \%)$ & 0.028 \\
\hline Stains & 920 (98.7\%) & 823 (98.9\%) & 97 (97.0\%) & 0.108 \\
\hline Thrombolysis & 37 (4.0\%) & 32 (3.9\%) & 5 (5.0\%) & 0.580 \\
\hline Stroke etiology & & & & $<0.001$ \\
\hline Atherosclerosis & 725 (77.9\%) & 658 (79.2\%) & 67 (67.0\%) & \\
\hline Cardioembolism & 94 (1.0\%) & $3(0.4 \%)$ & $0(0.0 \%)$ & \\
\hline Small vessel occlusion & 122 (13.1\%) & $94(11.3 \%)$ & $28(28.0 \%)$ & \\
\hline Other causes & $18(2.0 \%)$ & $17(2.0 \%)$ & $1(1.0 \%)$ & \\
\hline Length of hospital stay & $9.97(8.0-12.0)$ & $9.0(8.0-11.0)$ & $11.0(9.0-14.0)$ & $<0.001$ \\
\hline Discharge mRS score & $2.0(1.0-3.0)$ & $2.0(1.0-2.0)$ & $4.0(2.0-4.0)$ & $<0.001$ \\
\hline
\end{tabular}

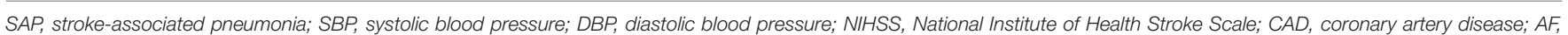
atrial fibrillation; mRS, modified Rankin Scale.

group (4.23 vs. $3.07 \mathrm{~g} / \mathrm{l}, 0.113$ vs. $0.087,13.85$ vs. $13.3 \mathrm{~s}, 1.08$ vs. $1.03,38.65$ vs. 36.70 s, 0.96 vs. $0.43 \mathrm{mg} / \mathrm{l}, P<0.001$, respectively), while albumin was much lower (36.45 vs. $38.1 \mathrm{~g} / \mathrm{l}, P<0.001)$, as shown in Figure 2.

\section{Baseline Characteristics of All Patients in the High and Low FAR Groups}

The ROC curve for FAR showed an AUC of 0.717 (95\% CI, $0.659-0.775 ; P<0.001)$ and pointed to an optimal cutoff level of $0.0977 \mathrm{mg} / \mathrm{dl}$ of FAR with $69.0 \%$ sensitivity and $66.1 \%$ specificity for the development of SAP during hospitalization (Figure 3). The patients were assigned to two groups [high FAR $(\geq 0.0977)$ or low FAR $(<0.0977)]$. The characteristics of the AIS participants between the two FAR groups, namely, the demographic, clinical, and laboratory characteristics, are depicted in Table 2. As shown in Table 2, patients with higher FAR levels were older; were more likely to be diabetic; had higher NIHSS scores at admission, length of stay, and mRS scores at discharge; and had higher fibrinogen, aPTT, D-dimer, and PLT counts, and lower albumin levels.

As shown in Table 3, after propensity score matching (PSM), 277 patients with high FAR levels were individually 1:1 matched to 277 patients with low FAR levels. The results above remained significant after controlling for the confounders, namely, fibrinogen, D-dimer, PLT counts, albumin, and the number of patients with SAP.

\section{Association Between the Level of FAR and SAP}

Table 4 showed the regression models for the association between FAR and SAP. After adjusting for potential confounders, FAR $\geq 0.0977$ was an independent predictive factor for higher risk of SAP [odds ratio (OR) 2.830; 95\% CI 1.654-4.840]. Moreover, age, aPTT, dysphagia, and NIHSS on admission were significantly associated with SAP in AIS patients (binary logistic regression, OR 1.031, 95\% CI 1.007-1.056; $P=0.011$; OR 1.074, 95\% CI 1.021-1.130; $P=0.005$; OR 5.216, 95\% CI 2.953-9.212; $P<0.001$; OR 1.138 95\% CI 1.076-1.203, respectively) (Table 4).

To assess the relationship between pneumonia severity and the FAR, we analyzed 100 patients with SAP (median FAR: $0.113[0.909-0.167])$. The high PSI group (0.13 [0.94-0.19] vs. 0.10 [0.79-0.12]; $P=0.008$ ) had significantly higher FARs than the low scoring groups, showing a close correlation between the FAR and pneumonia severity (Figure 4).

\section{DISCUSSION}

To the best of our knowledge, this is the first study to explore the role of FAR in the development of SAP. In this retrospective 


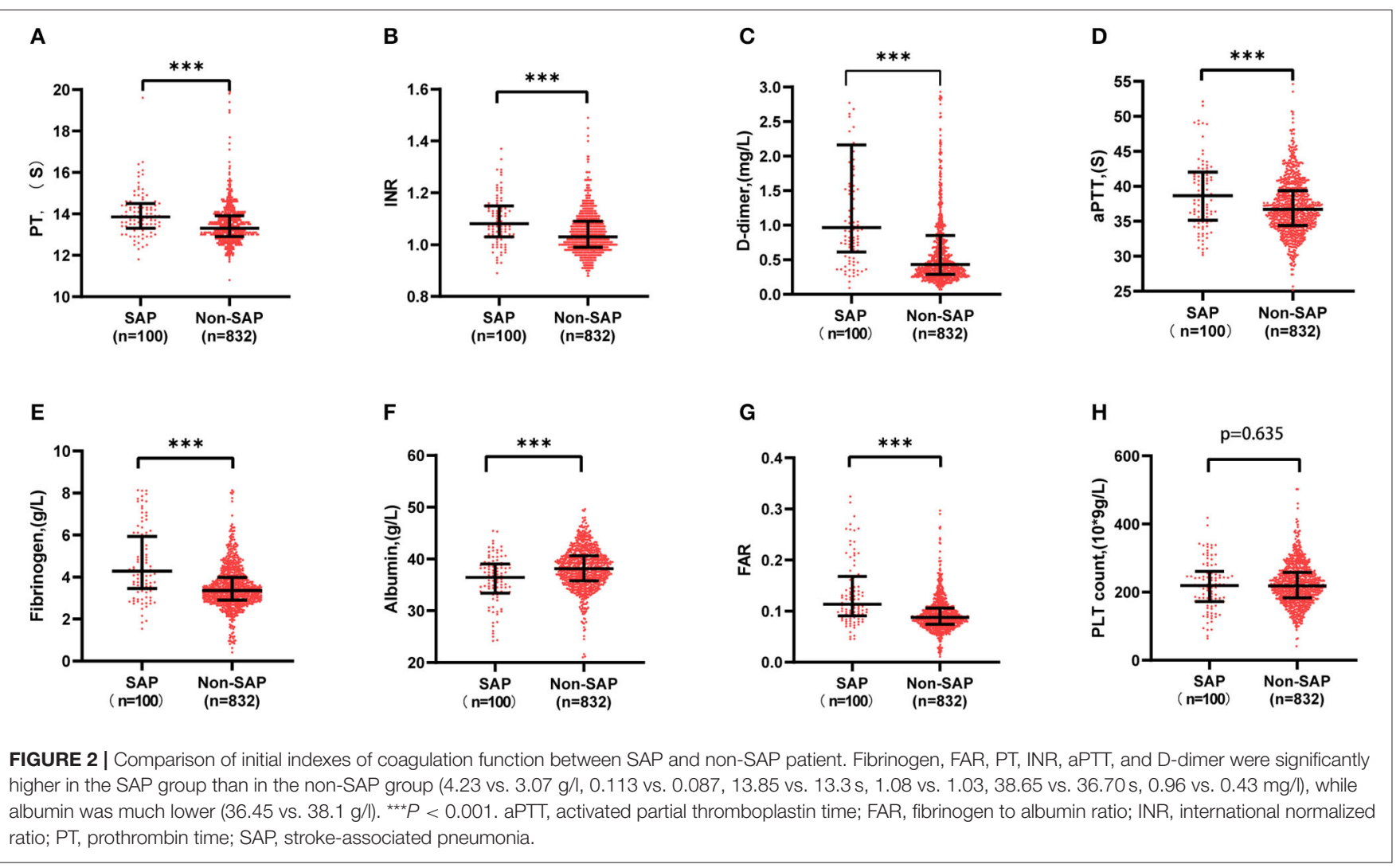

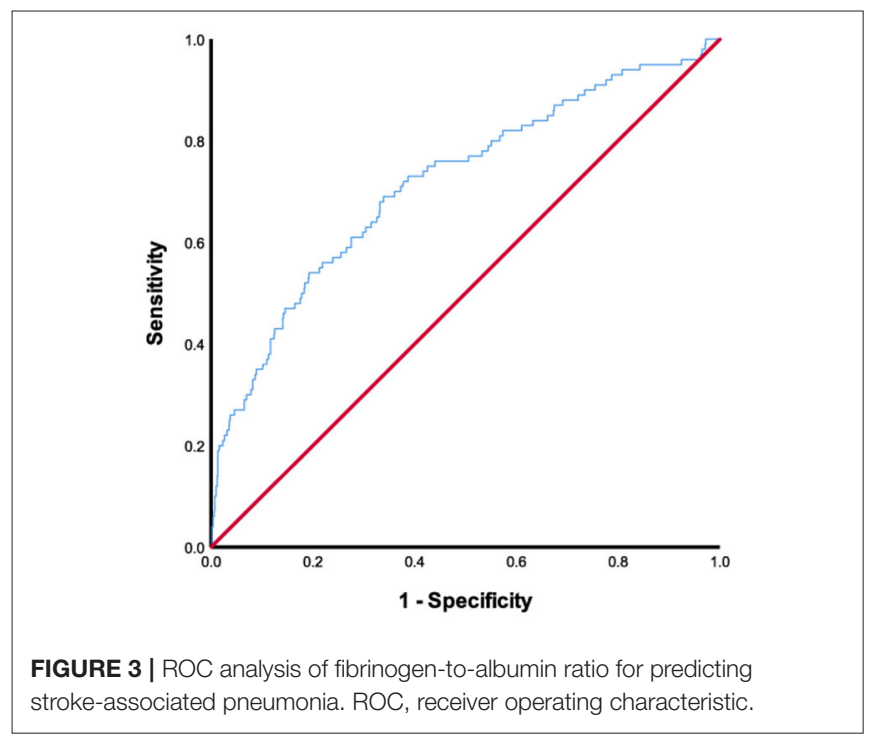

study, we found that coagulation functions differ significantly between patients with SAP and non-SAP. Patients with SAP had a higher level of fibrinogen, PT, aPTT, D-dimer, and lower albumin levels at admission. We also found an independent relationship between higher FAR in the first hours after hospitalization and the development of SAP after adjusting for potential contributory factors. This suggested the possible utility of FAR as a marker for the risk of developing SAP. In addition, high FAR levels correlated with pneumonia severity. Furthermore, our data demonstrated that FAR could effectively predict the incidence of SAP with an optimal cutoff point of 0.0977 that could identify the risk of SAP, with remarkable sensitivity and specificity. Therefore, FAR could be a useful and valid biomarker to identify potential patients with SAP at admission.

Compared with other literature, our study first described the coagulation parameters between SAP and non-SAP and found high FAR was an independent risk for the prevalence of SAP. In our study, 100 (10.7\%) patients were diagnosed with SAP, which was consistent with previous reports (6). The study showed age, baseline NIHSS score, AF, dysphagia, and length of hospital stay were contributory factors toward SAP, which was also consistent with previous reports (9). In addition, our study showed that antiplatelet therapy was related to a low risk of SAP. The link has not been studied yet. Studies regarding coagulation functions and SAP are rare. In an animal experiment carried out on models of acute lung injury, researchers found nebulized heparin could reduce pulmonary coagulopathy and inflammation effectively by modulating alveolar macrophages (31). During the COVID-19 pandemic, Bi et al. found that fibrinogen, FAR, and D-dimer were significantly elevated in severe patients with COVID-19 with inferior prognosis and accelerated clinical progression (32). A recent study found high levels of $\mathrm{D}$-dimer could increase the risk of 3-month mortality and death/severe disability in AF-related 
TABLE 2 | Baseline characteristics of all patients in the high $(\geq 0.0977)$ and low FAR $(<0.0977)$ groups.

\begin{tabular}{|c|c|c|c|c|}
\hline Variables & All patients & Low FAR $(n=580)$ & High FAR $(n=352)$ & $P$-value \\
\hline SAP & $100(10.7 \%)$ & $31(5.3 \%)$ & 69 (19.7\%) & $<0.001$ \\
\hline Age (years) & $67.0(59.0-74.0)$ & $65.0(57.0-72.0)$ & $69.0(62.0-77.0)$ & $<0.001$ \\
\hline Male & 596 (63\%) & 376 (64.7\%) & $220(62.7 \%)$ & 0.530 \\
\hline Current smoking & 376 (40.3\%) & 227 (39.1\%) & 149 (42.5\%) & 0.308 \\
\hline Drinking & 350 (37.6\%) & 232 (39.9\%) & 118 (33.6\%) & 0.054 \\
\hline NHISS & $3.0(1.0-6.0)$ & $2.0(1.0-5.0)$ & $4.0(1.0-8.0)$ & $<0.001$ \\
\hline Previous stroke & $133(14.3 \%)$ & 67 (11.5\%) & 65 (18.8\%) & 0.002 \\
\hline Hypertension & $713(76.5 \%)$ & 442 (76.1\%) & $271(77.2 \%)$ & 0.693 \\
\hline Diabetes mellitus & 369 (39.6\%) & 208 (35.8\%) & 161 (45.9\%) & 0.002 \\
\hline CAD & $20(2.2 \%)$ & $14(2.4 \%)$ & $6(1.7 \%)$ & 0.470 \\
\hline Anticoagulant drugs & $129(13.8 \%)$ & 72 (12.4\%) & 57 (16.4\%) & 0.099 \\
\hline Stains & 920 (98.7\%) & 575 (99.0\%) & 345 (98.3\%) & 0.375 \\
\hline Thrombolysis & 37 (4.0\%) & 30 (5.2\%) & 7 (2.0\%) & 0.016 \\
\hline Fibrinogen & $3.62(2.93-4.12)$ & $3.06(2.74-3.36)$ & $4.43(3.89-5.11)$ & $<0.001$ \\
\hline PT & $13.59(12.90-14)$ & $13.40(12.90-14.00)$ & $13.40(13.00-14.00)$ & 0.310 \\
\hline INR & $1.05(0.99-1.10)$ & $1.03(0.99-1.09)$ & $1.04(0.99-1.10)$ & 0.283 \\
\hline aPTा & 37.37 (34.50-39.80) & $36.2(34.30-38.8)$ & $37.5(34.8-40.92)$ & $<0.001$ \\
\hline D-dimer & $0.79(0.30-0.96)$ & $0.38(0.28-0.76)$ & $0.67(0.38-1.15)$ & $<0.001$ \\
\hline Albumin & 37.83 (35.50-40.50) & $39.10(37.00-41.60)$ & 35.70 (33.80-38.42) & $<0.001$ \\
\hline PLT & $218.0(181.0-258.0)$ & $214.0(180.0-248.0)$ & $226.0(186.0-273.0)$ & 0.001 \\
\hline Stroke etiology & & & & 0.247 \\
\hline
\end{tabular}

SAP, stroke-associated pneumonia; SBP, systolic blood pressure; DBP, diastolic blood pressure; INR, international normalized ratio; NIHSS, National Institute of Health Stroke Scale; CAD, coronary artery disease; AF, atrial fibrillation; PT, prothrombin time; aPTT, activated partial thromboplastin time; mRS, modified Rankin Scale.

patients with AIS (33). More evidence indicates that an extensive link exists between coagulation and inflammation. The acute phase of inflammation response leads to excessive activation of the coagulation system, and coagulation also amplifies the inflammation process considerably $(34,35)$. Despite this, the effect of FAR on acute patients with IS, of whom a high proportion suffer from coagulation dysfunction, had not been studied until now.

As fibrinogen and albumin, two critical factors in the coagulation system that care for biomarkers for nutrition state and inflammation, are delivered by the liver, the combined application of two factors (FAR) could be a more powerful predictor than single biomarker fibrinogen or albumin. An elevated FAR level may be a result of increased fibrinogen levels or/and decreased albumin levels. In this study, the FAR levels of patients with SAP were higher than patients with non-SAP ( 0.113 vs. $0.087, P<0.001$; Table 1$)$. The FAR level of patients with hemorrhagic transformation (HT) was higher than the patients with non-HT after stroke in a previous study (8.60 vs. 10.29, respectively; Table 1) (36). This may be due to the inflammatory response caused by the occurrence of stroke. When the immune system participates in the ischemic brain processes, stroke-induced immunodepression can protect our body from excessive inflammatory reactions. However, it promotes the risk of concurrent infections and affects the prognosis of stroke patients (37). Nonetheless, the association between FAR and AIS requires further study.

Fibrinogen, known as a $340 \mathrm{kDa}$ glycoprotein, can be deposited within and around damaged tissue zones. In research conducted in Spain, Miranda Acuña et al. found elevated fibrinogen deposition in the brain in a wide range of neurological diseases such as multiple sclerosis (38). However, the underlying mechanisms between fibrinogen and SAP remain unclear. There could be a potential mechanism to explain this association. 
TABLE 3 | Baseline characteristics of all patients in the high $(\geq 0.0977)$ and low FAR $(<0.0977)$ groups after propensity score matching.

\begin{tabular}{|c|c|c|c|c|}
\hline Variables & All patients & Low FAR $(n=277)$ & High FAR $(n=277)$ & $P$-value \\
\hline SAP & $67(12.1 \%)$ & $23(8.3 \%)$ & 44 (15.9\%) & 0.006 \\
\hline Age(years) & $68.0(61.0-76.0)$ & $69.0(62.0-75.0)$ & $68.0(61.0-76.0)$ & 0.644 \\
\hline Male & $211(38.1 \%)$ & 104 (37.5\%) & 107 (38.6\%) & 0.793 \\
\hline Current smoking & 218 (39.4\%) & 104 (37.5\%) & $114(41.2 \%)$ & 0.384 \\
\hline Drinking & $191(34.5 \%)$ & 94 (33.9\%) & 97 (35.0\%) & 0.789 \\
\hline Baseline SBP & 152 (137.0-168.0) & $155.0(139.0-169.0)$ & 151.0 (135.0-167.0) & 0.100 \\
\hline Baseline DBP & $82.0(74.0-91.0)$ & $82.0(75.0-92.0)$ & $82.0(73.0-91.0)$ & 0.677 \\
\hline NHISS & $3.0(1.0-7.0)$ & $3.0(1.0-7.0)$ & $3.0(1.0-7.0)$ & 0.331 \\
\hline Previous stroke & 82 (14.8\%) & $41(14.8 \%)$ & $41(14.8 \%)$ & 1.000 \\
\hline Hypertension & $416(75.1 \%)$ & 208 (75.1\%) & 208 (75.1\%) & 1.000 \\
\hline Diabetes mellitus & 226 (40.8\%) & 102 (36.8\%) & 124 (44.8\%) & 0.057 \\
\hline CAD & $13(2.4 \%)$ & $8(2.9 \%)$ & $5(1.8 \%)$ & 0.392 \\
\hline AF & $83(15.0 \%)$ & $41(14.8 \%)$ & 42 (15.2\%) & 0.905 \\
\hline Dysphagia & $521(94.0 \%)$ & 260 (93.9\%) & $261(94.2 \%)$ & 0.858 \\
\hline Antiplatelet drugs & $82(14.8 \%)$ & 40 (14.4\%) & 42 (15.2\%) & 0.811 \\
\hline Anticoagulant drugs & $546(98.6 \%)$ & 275 (99.3\%) & $271(97.8 \%)$ & 0.154 \\
\hline Stains & $18(3.2 \%)$ & $11(4.0 \%)$ & 7 (2.5\%) & 0.338 \\
\hline Thrombolysis & $84(15.2 \%)$ & $43(15.5 \%)$ & $41(14.8 \%)$ & 0.813 \\
\hline Fibrinogen & $3.63(3.08-4.35)$ & $3.1(2.8-3.41)$ & 4.35 (3.86-5.01) & $<0.001$ \\
\hline PT & $13.4(13.0-14.0)$ & 13.5 (12.9-14.0) & $13.4(13.0-13.9)$ & 0.705 \\
\hline INR & $1.04(1.00-1.10)$ & $1.04(1.00-1.10)$ & 1.04 (0.99-1.09) & 0.696 \\
\hline APTT & 36.95 (34.6-40.0) & 36.5 (34.7-39.8) & $37.2(34.5-40.4)$ & 0.456 \\
\hline D-dimer & $0.51(0.31-0.97)$ & $0.43(0.28-0.76)$ & $0.61(0.36-1.06)$ & $<0.001$ \\
\hline Albumin & 37.50 (35.10-40.10) & $38.90(36.80-41.30)$ & 36.00 (34.00-38.95) & $<0.001$ \\
\hline PLT & $227.0(192.0-265.2)$ & 233.0 (198.0-266.5) & 218 (181.0-263.0) & 0.026 \\
\hline Stroke etiology & & & & 0.818 \\
\hline Atherosclerosis & $421(76.1 \%)$ & 213 (76.9\%) & 208 (75.4\%) & \\
\hline Cardioembolism & 88 (15.9\%) & 43 (15.5\%) & 45 (16.3\%) & \\
\hline Small vessel occlusion & $34(6.1 \%)$ & $16(5.8 \%)$ & $18(6.5 \%)$ & \\
\hline Other causes & $10(3.8 \%)$ & $5(1.9 \%)$ & 5 (1.9\%) & \\
\hline Length of hospital stay & $9(8.0-12.0)$ & $9.0(8.0-12.0)$ & $9.0(8.0-12.0)$ & 0.555 \\
\hline Discharge mRS score & $2.0(1.0-3.0)$ & $2.0(1.0-3.0)$ & $2.0(1.0-3.0)$ & 0.492 \\
\hline
\end{tabular}

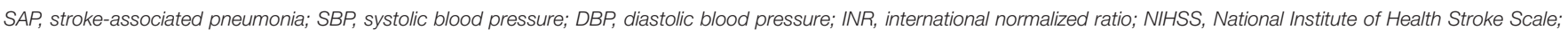
CAD, coronary artery disease; AF, atrial fibrillation; PT, prothrombin time; aPTT, activated partial thromboplastin time; mRS, modified Rankin Scale.

First, fibrinogen is identified as a ligand for various cell surface receptors. It could serve as an intermediate molecule to boost cell-to-cell adhesion between leukocytes and the endothelium. In this way, fibrinogen can facilitate the migration of leukocytes outside the blood vessel to specific tissues $(39,40)$, and induce changes in leukocytes function. This can take place through cell movement, phagocytosis, release of chemokines and cytokines, degranulation, and nuclear factor-Kappa Bmediated transcription (41-45). Second, plasma fibrinogen levels increase by $2-3$ times in response to inflammation, causing cell aggregation, higher blood viscosity, and an increase in endothelin-1 (46). Moreover, during the fibrinolysis process, plasmin, the major fibrinolytic protease, can degrade extracellular matrix proteins and activates matrix metalloproteinases resulting in tissue damage $(47,48)$.

Serum albumin levels represent the state of nutrition status of an individual and indicate the presence of inflammation. Inflammation increases capillary permeability and flee of serum albumin, causing the distribution volume of albumin to increase while total albumin mass decreases. Albumin accumulates at inflammation sites so it has been widely used as an ideal drug delivery platform in antiinflammation therapy (49). Several studies revealed that low serum albumin was considered a high-risk factor for deterioration and poor prognosis in patients with nosocomial infection (50, 51). Growing research evidence showed that albumin was a useful, independent prognostic factor in patients after stroke $(52,53)$. In a cohort study of 759 patients with AIS followed for 3 months, a poor outcome was independently related to low serum albumin level, ischemic heart disease, and infarction size (53). Based on physiological characteristics of albumin, namely, antioxidant, anti-inflammatory, anticoagulant, and antiplatelet aggregation activity and regulation of microvascular permeability, albumin could be used to predict the pulmonary infection after stroke. 
TABLE 4 | Multivariate logistic models for risk factors of SAP.

\begin{tabular}{|c|c|c|c|c|}
\hline & Unadjusted analysis & & Adjusted analysis & \\
\hline & OR (95\% Cl) & $P$-value & OR $(95 \% \mathrm{Cl})$ & $P$-value \\
\hline Age & 1.059 (1.037-1.081) & $<0.001$ & $1.031(1.007-1.056)$ & 0.011 \\
\hline Sex, female & 1.047 (0.681-1.609) & 0.834 & & \\
\hline NIHSS on admission & 1.251 & $<0.001$ & 1.138 (1.076-1.203) & $<0.001$ \\
\hline Atrial fibrillation & 3.308 (2.038-5.370) & $<0.001$ & NS & NS \\
\hline Dysphagia & 12.520 (7.898-19.845) & $<0.001$ & $5.216(2.953-9.212)$ & $<0.001$ \\
\hline Albumin $^{a}$ & $0.886(0.848-0.927)$ & $<0.001$ & & \\
\hline PT & $1.279(1.111-1.427)$ & 0.001 & NS & NS \\
\hline INR & 10.285 (2.609-40.554) & 0.001 & NS & NS \\
\hline aPTा & $1.102(1.059-1.146)$ & $<0.001$ & $1.074(1.021-1.130)$ & 0.005 \\
\hline Fibrinogen $^{a}$ & 1.959 (1.668-2.300) & $<0.001$ & & \\
\hline D-dimer & $1.065(1.022-1.011)$ & $<0.001$ & NS & NS \\
\hline High FAR (FAR $\geq 0.0977)$ & $4.341(2.775-6.791)$ & $<0.001$ & $2.830(1.654-4.840)$ & $<0.001$ \\
\hline Anticoagulant drugs & $1.782(1.057-3.003)$ & 0.03 & NS & NS \\
\hline Antiplatelet drugs & $0.241(0.126-0.461)$ & $<0.001$ & NS & NS \\
\hline Length of stay & 1.137 (1.083-1.193) & $<0.001$ & NS & NS \\
\hline
\end{tabular}

Logistic regression analysis adjusted for the 12 variables $(P<0.1)$ listed. SAP, stroke-associated pneumonia; OR, odds radio; FAR, fibrinogen to albumin ratio; PT, prothrombin time; aPTT, activated partial thromboplastin time; INR, international normalized ratio.

a These parameters are not entered in the multivariate analysis in order to prevent multicollinearity.

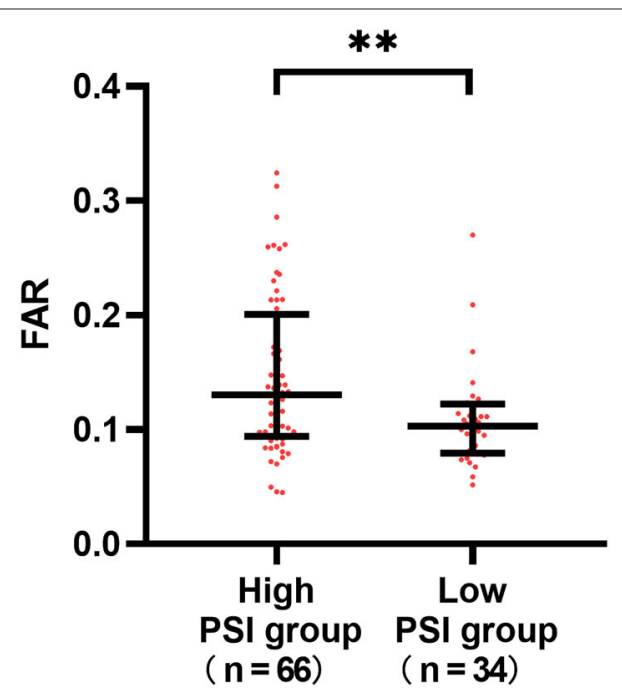

FIGURE 4 | Differences in the fibrinogen to albumin (FAR) according to the severity of stroke-associated pneumonia (SAP). The high PSI group $(0.13$ $[0.94-0.19]$ vs. $0.10[0.79-0.12] ; P=0.008)$ had significantly higher FARs than the low scoring groups. ${ }^{\star \star} P<0.01$.

Notably, FAR baseline levels correlated with poststroke pneumonia during hospitalization. It also seems plausible that there may be an association between FAR and pneumonia severity, although inflammation response changes dynamically overtime during AIS. It is interesting that this relationship continued despite the effects of the inflammation process.
There remained some limitations in our study. First, our study was based on a single-center retrospective database and some inevitable subjective selections bias might have existed, reducing the reliability of the investigation. Second, FAR was only recorded once at admission and could not be recorded at other times (beyond $24 \mathrm{~h}$ ), which limited the analysis of the dynamic association between FAR and SAP. It is necessary to conduct a further longitudinal study to verify the predictive value of FAR measured at multiple times for SAP. Third, our study lacked data on the use of the nasogastric tubes and mechanical ventilation, which were related to a high risk of respiratory infections. Finally, other important blood parameters such as antithrombin and leukocyte were not included in this study.

\section{CONCLUSIONS}

Despite the limitations, our findings are very meaningful. Our study revealed that high FAR was an independent predictive factor related to a higher risk of SAP in patients with AIS. It was also related to the severity of pneumonia. The study provides new references for further clinical trials to determine whether the levels of FAR may serve as a new marker to select patients with AIS at a higher risk of SAP and thus, to guide targeted treatment at an early stage.

\section{DATA AVAILABILITY STATEMENT}

The original contributions presented in the study are included in the article/supplementary 
material, further inquiries can be directed to the corresponding author/s.

\section{ETHICS STATEMENT}

The studies involving human participants were reviewed and approved by the Ethics Committee of First Affiliated Hospital of Wenzhou Medical University. Written informed consent for participation was not required for this study in accordance with the national legislation and the institutional requirements.

\section{AUTHOR CONTRIBUTIONS}

GL, MH, JS, ZW, and JH conceived and designed the study. GL and $\mathrm{MH}$ interpreted data. GL, MH, and JS wrote the manuscript. GL, JS, and XX prepared figures. GL and JS did the statistical analyses. GL, MH, JS, XX, HL, LQ, HZ, MX, DG, and LY

\section{REFERENCES}

1. Kumar S, Selim MH, Caplan LR. Medical complications after stroke. Lancet Neurol. (2010) 9:105-18. doi: 10.1016/s1474-4422(09)70266-2

2. Tong X, Kuklina EV, Gillespie C, George MG. Medical complications among hospitalizations for ischemic stroke in the United States from 1998 to 2007. Stroke. (2010) 41:980-6. doi: 10.1161/strokeaha.110.578674

3. Wang YJ Li ZX, Gu HQ, Zhai Y, Jiang Y, Zhao XQ, et al. China Stroke Statistics 2019: a report from the National Center for Healthcare Quality Management in Neurological Diseases, China National Clinical Research Center for Neurological Diseases, the Chinese Stroke Association, National Center for Chronic and Non-communicable Disease Control and Prevention, Chinese Center for Disease Control and Prevention and Institute for Global Neuroscience and Stroke Collaborations. Stroke Vasc Neurol. (2020) 5:211-39. doi: 10.1136/svn-2020-000457

4. Smith CJ, Bray BD, Hoffman A, Meisel A, Heuschmann PU, Wolfe CD, et al. Can a novel clinical risk score improve pneumonia prediction in acute stroke care? A UK multicenter cohort study. J Amer Heart Assoc. (2015) 4:e001307. doi: $10.1161 /$ jaha.114.001307

5. Sui R, Zhang L. Risk factors of stroke-associated pneumonia in Chinese patients. Neurol Res. (2011) 33:508-13. doi: 10.1179/016164111x13007856084205

6. Badve MS, Zhou Z, van de Beek D, Anderson CS, Hackett ML. Frequency of post-stroke pneumonia: systematic review and meta-analysis of observational studies. Int J Stroke. (2019) 14:125-36. doi: 10.1177/1747493018806196

7. Ingeman A, Andersen G, Hundborg HH, Svendsen ML, Johnsen SP. Inhospital medical complications, length of stay, and mortality among stroke unit patients. Stroke. (2011) 42:3214-8. doi: 10.1161/strokeaha.110.610881

8. Katzan IL, Cebul RD, Husak SH, Dawson NV, Baker DW. The effect of pneumonia on mortality among patients hospitalized for acute stroke. Neurology. (2003) 60:620-5. doi: 10.1212/01.wnl.0000046586.38284.60

9. Finlayson O, Kapral M, Hall R, Asllani E, Selchen D, Saposnik G. Risk factors, inpatient care, and outcomes of pneumonia after ischemic stroke. Neurology. (2011) 77:1338-45. doi: 10.1212/WNL.0b013e31823152b1

10. Ji R, Wang D, Shen H, Pan Y, Liu G, Wang P, et al. Interrelationship among common medical complications after acute stroke: pneumonia plays an important role. Stroke. (2013) 44:3436-44. doi: 10.1161/strokeaha.113.001931

11. Vermeij JD, Westendorp WF, Dippel DW, van de Beek D, Nederkoorn PJ. Antibiotic therapy for preventing infections in people with acute stroke. Cochrane Database Syst Rev. (2018) 1:Cd008530. doi: 10.1002/14651858.CD008530.pub3

12. Kalra L, Irshad S, Hodsoll J, Simpson M, Gulliford M, Smithard D, et al. Prophylactic antibiotics after acute stroke for reducing pneumonia in patients screened and extracted data. $\mathrm{ZW}, \mathrm{JH}$, and GH supervised the study. All authors have made an intellectual contribution to the manuscript and approved the submission.

\section{FUNDING}

This work was supported by the Projects of Provincial Natural Science Foundation of Zhejiang (no. LY19H090013), the Jiaxing Science and Technology Plan Project (no. 2019AD32181) and Institute of Aging, Key Laboratory of Alzheimer's Disease of Zhejiang Province, Wenzhou Medical University, Wenzhou, Zhejiang, China.

\section{ACKNOWLEDGMENTS}

We thank the study participants and the clinical staff at all participating hospitals for their support and contribution to this project.

with dysphagia (STROKE-INF): a prospective, cluster-randomised, openlabel, masked endpoint, controlled clinical trial. Lancet. (2015) 386:1835-44. doi: 10.1016/s0140-6736(15)00126-9

13. Zapata-Arriaza E, Mancha F, Bustamante A, Moniche F, Pardo-Galiana B, Serrano-Gotarredona $\mathrm{P}$, et al. Biomarkers predictive value for early diagnosis of stroke-associated pneumonia. Ann Clin Transl Neurol. (2019) 6:1882-7. doi: 10.1002/acn3.50849

14. Hoffmann S, Malzahn U, Harms H, Koennecke HC, Berger K, Kalic M, et al. Development of a clinical score (A2DS2) to predict pneumonia in acute ischemic stroke. Stroke. (2012) 43:2617-23. doi: 10.1161/strokeaha.112.653055

15. Ji R, Shen H, Pan Y, Wang P, Liu G, Wang Y, et al. Novel risk score to predict pneumonia after acute ischemic stroke. Stroke. (2013) 44:1303-9. doi: 10.1161/strokeaha.111.000598

16. Harms H, Grittner U, Dröge H, Meisel A. Predicting post-stroke pneumonia: the PANTHERIS score. Acta Neurol Scand. (2013) 128:178-84. doi: 10.1111/ane.12095

17. Friedant AJ, Gouse BM, Boehme AK, Siegler JE, Albright KC, Monlezun DJ, et al. A simple prediction score for developing a hospital-acquired infection after acute ischemic stroke. J Stroke Cerebrovasc Dis. (2015) 24:6806. doi: 10.1016/j.jstrokecerebrovasdis.2014.11.014

18. Helmy TA, Abd-Elhady MA, Abdou M. Prediction of ischemic strokeassociated pneumonia: a comparison between 3 scores. J Stroke Cerebrovasc Dis. (2016) 25:2756-61. doi: 10.1016/j.jstrokecerebrovasdis.2016.07.030

19. Gong S, Zhou Z, Zhou M, Lei Z, Guo J, Chen N, et al. Validation of risk scoring models for predicting stroke-associated pneumonia in patients with ischaemic stroke. Stroke Vasc Neurol. (2016) 1:122-6. doi: 10.1136/svn-2016-0 00025

20. Nam KW, Kim TJ, Lee JS, Kwon HM, Lee YS, Ko SB, et al. High neutrophilto-lymphocyte ratio predicts stroke-associated pneumonia. Stroke. (2018) 49:1886-92. doi: 10.1161/strokeaha.118.021228

21. Frantzeskaki F, Armaganidis A, Orfanos SE. Immunothrombosis in acute respiratory distress syndrome: cross talks between inflammation and coagulation. Respiration. (2017) 93:212-25. doi: 10.1159/000453002

22. Zheng L, Wang Z, Liu J, Yang X, Zhang S, Hao Z, et al. Association between admission blood fibrinogen-to-albumin ratio and clinical outcomes after acute lacunar stroke. Biomark Med. (2021) 15:87-96. doi: 10.2217/bmm-2019-0537

23. An Q, Liu W, Yang Y, Yang B. Preoperative fibrinogen-to-albumin ratio, a potential prognostic factor for patients with stage IB-IIA cervical cancer. BMC Cancer. (2020) 20:691. doi: 10.1186/s12885-020-07191-8

24. He ZQ, Duan H, Lin FH, Zhang J, Chen YS, Zhang GH, et al. Pretreatment neutrophil-to-lymphocyte ratio plus albumin-to-gamma-glutamyl transferase 
ratio predict the diagnosis of grade III glioma. Ann Transl Med. (2019) 7:623. doi: 10.21037/atm.2019.11.24

25. Li M, Tang C, Luo E, Qin Y, Wang D, Yan G. Relation of fibrinogen-toalbumin ratio to severity of coronary artery disease and long-term prognosis in patients with non-ST elevation acute coronary syndrome. Biomed Res Int. (2020) 2020:1860268. doi: 10.1155/2020/1860268

26. Fine MJ, Auble TE, Yealy DM, Hanusa BH, Weissfeld LA, Singer $\mathrm{DE}$, et al. A prediction rule to identify low-risk patients with community-acquired pneumonia. $N$ Engl J Med. (1997) 336:243-50. doi: 10.1056/nejm199701233360402

27. Aujesky D, Fine MJ. The pneumonia severity index: a decade after the initial derivation and validation. Clin Infect Dis. (2008) 47 Suppl 3:S133-9. doi: $10.1086 / 591394$

28. Brodsky MB, Suiter DM, González-Fernández M, Michtalik HJ, Frymark TB, Venediktov R, et al. Screening accuracy for aspiration using bedside water swallow tests: a systematic review and meta-analysis. Chest. (2016) 150:148-63. doi: 10.1016/j.chest.2016.03.059

29. Shah BA, Ahmed W, Dhobi GN, Shah NN, Khursheed SQ, Haq I. Validity of pneumonia severity index and CURB-65 severity scoring systems in community acquired pneumonia in an Indian setting. Indian J Chest Dis Allied Sci. (2010) 52:9-17.

30. Smith CJ, Kishore AK, Vail A, Chamorro A, Garau J, Hopkins SJ, et al. Diagnosis of stroke-associated pneumonia: recommendations from the pneumonia in stroke consensus group. Stroke. (2015) 46:2335-40. doi: 10.1161/strokeaha.115.009617

31. Chimenti L, Camprubí-Rimblas M, Guillamat-Prats R, Gomez MN, Tijero J, Blanch L, et al. Nebulized heparin attenuates pulmonary coagulopathy and inflammation through alveolar macrophages in a rat model of acute lung injury. Thromb Haemost. (2017) 117:2125-34. doi: 10.1160/th17-05-0347

32. Bi X, Su Z, Yan $H$, Du J, Wang J, Chen L, et al. Prediction of severe illness due to COVID-19 based on an analysis of initial fibrinogen to albumin ratio and platelet count. Platelets. (2020) 31:674-9. doi: 10.1080/09537104.2020.1760230

33. Yang X, Lu T, Qu Z, Zhang Y, Liu P, Ma Y. Plasma D-dimer level is associated with clinical outcomes in patients with atrial fibrillation related acute ischemic stroke after pneumonia. BMC Neurol. (2021) 21:137. doi: 10.1186/s12883-021-02168-x

34. Levi M, van der Poll T. Inflammation and coagulation. Crit Care Med. (2010) 38(2 Suppl):S26-34. doi: 10.1097/CCM.0b013e3181c98d21

35. Foley JH, Conway EM. Cross talk pathways between coagulation and inflammation. Circ Res. (2016) 118:1392-408. doi: 10.1161/circresaha.116.306853

36. Ruan Y, Yuan C, Liu Y, Zeng Y, Cheng H, Cheng Q, et al. High fibrinogen-to-albumin ratio is associated with hemorrhagic transformation in acute ischemic stroke patients. Brain Behav. (2021) 11:e01855. doi: 10.1002/brb3.1855

37. Liu DD, Chu SF, Chen C, Yang PF, Chen NH, He X. Research progress in stroke-induced immunodepression syndrome (SIDS) and stroke-associated pneumonia (SAP). Neurochem Int. (2018) 114:42-54. doi: 10.1016/j.neuint.2018.01.002

38. Miranda Acuña J, Hidalgo de la Cruz M, Ros AL, Tapia SP, Martínez Ginés ML, de Andrés Frutos CD. Elevated plasma fibrinogen levels in multiple sclerosis patients during relapse. Multi Scler Relat Disord. (2017) 18:157-60. doi: 10.1016/j.msard.2017.09.033

39. Yakovlev S, Medved L. Effect of fibrinogen, fibrin, and fibrin degradation products on transendothelial migration of leukocytes. Thromb Res. (2018) 162:93-100. doi: 10.1016/j.thromres.2017.11.007

40. Yakovlev S, Mikhailenko I, Cao C, Zhang L, Strickland DK, Medved L. Identification of VLDLR as a novel endothelial cell receptor for fibrin that modulates fibrin-dependent transendothelial migration of leukocytes. Blood. (2012) 119:637-44. doi: 10.1182/blood-2011-09-382580

41. Tuluc F, Garcia A, Bredetean O, Meshki J, Kunapuli SP. Primary granule release from human neutrophils is potentiated by soluble fibrinogen through a mechanism depending on multiple intracellular signaling pathways. Amer J Physiol Cell Physiol. (2004) 287:C1264-72. doi: 10.1152/ajpcell.00177.2004

42. Rubel C, Gómez S, Fernández GC, Isturiz MA, Caamaño J, Palermo MS. Fibrinogen-CD11b/CD18 interaction activates the NF-kappa B pathway and delays apoptosis in human neutrophils. Eur J Immunol. (2003) 33:1429-38. doi: 10.1002/eji.200323512

43. Smiley ST, King JA, Hancock WW. Fibrinogen stimulates macrophage chemokine secretion through toll-like receptor 4. J Immunol. (2001) 167:2887-94. doi: 10.4049/jimmunol.167.5.2887

44. Sitrin RG, Pan PM, Srikanth S, Todd RF, III. Fibrinogen activates NFkappa B transcription factors in mononuclear phagocytes. J Immunol. (1998) 161:1462-70.

45. Rubel C, Fernández GC, Dran G, Bompadre MB, Isturiz MA, Palermo MS. Fibrinogen promotes neutrophil activation and delays apoptosis. J Immunol. (2001) 166:2002-10. doi: 10.4049/jimmunol.166.3.2002

46. Sen U, Tyagi N, Patibandla PK, Dean WL, Tyagi SC, Roberts AM, et al. Fibrinogen-induced endothelin-1 production from endothelial cells. Amer J Physiol Cell Physiol. (2009) 296:C840-7. doi: 10.1152/ajpcell.0051 5.2008

47. Plow EF, Herren T, Redlitz A, Miles LA, Hoover-Plow JL. The cell biology of the plasminogen system. FASEB J. (1995) 9:939-45. doi: 10.1096/fasebj.9.10.7615163

48. Monea S, Lehti K, Keski-Oja J, Mignatti P. Plasmin activates pro-matrix metalloproteinase-2 with a membrane-type 1 matrix metalloproteinase-dependent mechanism. J Cell Physiol. (2002) 192:160-70. doi: $10.1002 /$ jcp.10126

49. Sleep D. Albumin and its application in drug delivery. Expert Opin Drug Deliv. (2015) 12:793-812. doi: 10.1517/17425247.2015.9 93313

50. Dziedzic T, Pera J, Klimkowicz A, Turaj W, Slowik A, Rog TM, et al. Serum albumin level and nosocomial pneumonia in stroke patients. Eur J Neurol. (2006) 13:299-301. doi: 10.1111/j.1468-1331.2006.0 1210.x

51. Domínguez de. Villota E, Mosquera JM, Rubio JJ, Galdos P, Díez Balda V, de la Serna JL, et al. Association of a low serum albumin with infection and increased mortality in critically ill patients. Intensive Care Med. (1980) 7:19-22. doi: 10.1007/bf01692917

52. Idicula TT, Waje-Andreassen U, Brogger J, Naess H, Thomassen L. Serum albumin in ischemic stroke patients: the higher the better. The Bergen Stroke Study. Cerebrovasc Dis. (2009) 28:13-7. doi: 10.1159/0002 15938

53. Seet RC, Lim EC, Chan BP, Ong BK. Serum albumin level as a predictor of ischemic stroke outcome. Stroke. (2004) 35:2435; author reply -6. doi: 10.1161/01.Str.0000145487.89910.12

Conflict of Interest: The authors declare that the research was conducted in the absence of any commercial or financial relationships that could be construed as a potential conflict of interest.

Publisher's Note: All claims expressed in this article are solely those of the authors and do not necessarily represent those of their affiliated organizations, or those of the publisher, the editors and the reviewers. Any product that may be evaluated in this article, or claim that may be made by its manufacturer, is not guaranteed or endorsed by the publisher.

Copyright (C) $2022 \mathrm{Lin}, \mathrm{Hu}$, Song, Xu, Liu, Qiu, Zhu, Xu, Geng, Yang, Huang, He and Wang. This is an open-access article distributed under the terms of the Creative Commons Attribution License (CC BY). The use, distribution or reproduction in other forums is permitted, provided the original author(s) and the copyright owner(s) are credited and that the original publication in this journal is cited, in accordance with accepted academic practice. No use, distribution or reproduction is permitted which does not comply with these terms. 